\title{
Minor Surgical Procedures in Children
}

JAMES E. RASMUSSEN, M.D.

I do not function primarily as a surgeon, pediatrician, or psychiatrist. I do, however, have considerable experience with minor surgery in children and am pleased to have this opportunity to share my approach with you. The reader will quickly note the paucity of references, reflecting in part the dearth of suitable sources and in part my own personal preferences. Also appreciate that rarely is a technique for calming anxiety in children "right or wrong." You should use procedures that produce as little emotional and physical disturbance as possible and that are consistent with good surgical practices.

\section{THE PATIENT AS A WHOLE}

Whether the patient needs a simple punch biopsy or removal of an extensive congenital nevus do not forget that kids are people too, and people are not simply the moles or hemangiomas or other dermatoses presented to you. In some cases, cutaneous disease and its treatment may be associated with far more significant systemic problems, such as epilepsy or mental retardation. Most of these diseases are no different than those found in adults, but some have the potential to adversely affect growth and development of a child (e.g., epidermal nevi, neurofibromatosis, tuberous sclerosis, xeroderma pigmentosa). The dermatologist will usually be informed by the mother or the child's pediatrician or family practitioner if a patient's problems are more than skin deep since, in most cases, the dermatologist is not the primary-care physician.

However, this may not be true for all patients, and if there is any reason to suspect growth retardation, height and weight should be measured and compared to normals. These standardized milestones of growth and development can be found in the "Harriet Lane Handbook" (Year Book Medical). Many physicians make the argument that these measurements should be obtained on every patient under the age of 15 , but this is generally unnecessary for the dermatologist because the patient's pediatrician will surely have that information. In cases of systemic disease or lack of a pediatri-

Dr. Rasmussen is Associate Professor of Dermatology and Pediatrics, University of Michigan Medical School, Ann Arbor, Michigan. cian, these measurements should be taken because they may be useful for comparison with previous visits or in long-term follow-up.

\section{THE PATIENT AND THE IMPENDING HOLE}

Because children younger than 9 to 12 months old cannot comprehend a procedural explanation, your description of the operation should be directed entirely to the parents. One- to 2-year-olds can comprehend, and may obey, simple commands such as lie down and hold still. Very short explanations are appropriate in this age group but do not waste any words. From 2 through 7 or 8 years most children appreciate simple explanations and usually follow commands. Beyond 8 years pediatric patients can usually be treated as adults-Explanations always are appropriate and restraints or sedation rarely needed.

Children from 6 months to 6 years commonly cry when seeing a physician, partly because of the "strange" face, parental pre-conditioning, or previously traumatic visits to doctors. I have not found any easy solution to this problem; children instinctively know that crying is their only weapon. The following suggestions may be helpful, however.

1. From 6 months through 4 to 5 years most children have a considerable fear of separation from the parent and it is usually best to have one or both in attendance. Before or after this age a decision on whom to allow in the room will usually depend on the patient, procedure, parents, and surgeon. I prefer that a parent observe most dermatologic surgery.

2. Don't tower over the patient more than is necessary. Move four to five feet away during the interview and after the exam, which should be conducted in the parent's lap if at all possible. A thorough physical examination is impossible, however, unless the patient is flat on the exam table. Keep this upsetting time to the minimum possible but do not cut corners and make mistakes.

3. Move slowly and describe what you are going to do. Sudden, sharp movements, especially if directed toward the patient's face, usually provoke considerable anxiety.

4. White coats are alright for some kids but not for others. In my experience there is no association between fright and white. Once the child starts crying, 
simply taking off a laboratory coat rarely stops the outburst, and soothing words rarely stop the noise.

5. Never rush a procedure to placate a crying child. You frequently wind up with an inadequate biopsy which then must be repeated. Crying should motivate you to do it so well that the patient does not need to return.

6. Don't take it personally.

\section{SEDATION VERSUS RESTRAINT}

Children younger than 7 to 8 years of age rarely submit to a surgical procedure without resistance. Possible approaches include sedation, restraint, or referral to a general surgeon. Some psychiatrists and other physicians believe that "only in dire circumstances should immobilization by wrapping be used," preferring general anesthesia or "cocktails" instead. These mixtures frequently contain demerol-phenergan and thorazine (DPT) and are used on a milligram per kilogram basis. Valium is also commonly used as a single sedative agent. My objections to sedation are that paradoxical reactions (agitation, hallucination), although uncommon, occur in about $1 \%$ to $4 \%$ of cases and are terribly frightening to patient, parents, and physician; inadequate response is common in about half of all pediatric cases and restraints are still necessary (this is especially true in mentally retarded or emotionally disturbed patients); and, although rare, respiratory depression sometimes occurs, necessitating the availability of resuscitation and ventilation equipment.

Consequently I much prefer restraints that are never physically harmful. Even though some contend that these physical restraints can damage the psyche, I have yet to see a significant immediate or delayed reaction to them. Immobilization can best be accomplished with firm pressure from parents and assistants unless the procedure will take longer than a few minutes, in which case a "papoose board" (Olympic Surgical Company, small and large sizes) is most appropriate. The cleverly arranged system of straps is not painful and yet quite effective. An extremity can be immobilized with gauze and tape on an arm board.

Hypnosis. In the hands of a trained operator hypnosis provides a rapid method of sedation and relaxation which is very useful in children. The American Society of Clinical Hypnosis (2400 East Devon Avenue, Suite 218, Des Plaines, Illinois 60018) presents several workshops a year throughout the United States. These sessions are directed exclusively toward the medical profession-physicians, dentists, psychol- ogists, etc. With study and practice hypnosis can be a rapid means to induce surgical relaxation and sedation in young children, although it is usually not effective for deep local anesthesia.

Nitrous Oxide. Several recent articles have suggested that use of nitrous oxide is a reasonable approach to sedation or anesthesia. ${ }^{2-4}$ The ease of application and relative safety make this technique attractive. However, the potential for a medical disaster is real because anoxia and aspiration may occur; proper training in administration and resuscitation is essential and fool-proof "fail-safe" systems are a must.

\section{POSTOPERATIVE CARE}

There is no "child-proof" dressing. Most children do not usually insist on removing bandages but if this appears to be the case either do without or use a wrap or spray if possible. Also remember that if the sutures were difficult to insert-crying child, anxious parentthey will also be hard to remove. Consider absorbable sutures in this situation. Wounds should be cleansed daily. Hydrogen peroxide or plain soap and water will usually suffice, with a thin film of ointment (i.e., Bacitracin) covering the wound.

In my experience children are remarkably more tolerant of postoperative pain than adults. I rarely find it necessary to prescribe analgesics other than acetominophen.

There are a number of performances that will make surgery more memorable than haunting: Blow up a clean glove and paint a face on it, give a small, safe toy as a gift, pour liquid nitrogen down the drain followed by a stream of water. Another technique is to paint a face on the wound using the suture line as a mouth or a nose. It sometimes is difficult, however, to determine the audience that most appreciates these maneuvers. Occasionally I feel that the parents and physician get more from this than the children.

\section{REFERENCES}

1. Gabriel, H. P. A practical approach to preparing children for dermatologic surgery. 1. Dermatol. Surg. Oncol. 3:523-526, 1977.

2. Maloney, J. M. Nitrous oxide-oxygen analgesia in dermatologic surgery. J. Dermatol. Surg. Oncol. 6:447-450, 1980.

3. Maloney, J. M., Coleman, W. P., and Mora, R. Analgesia induced by nitrous oxide and oxygen as an adjunct to local anesthesia in dermatologic surgery: Results of clinical trials. J. Dermatol. Surg. Oncol. 6:939-943, 1980.

4. Griffin, G. C., Campbell, V. D., and Jones, R. Nitrous oxide-oxygen sedation for minor surgery: Experience in a pediatric setting. J.A.M.A. 245:2411-2413, 1981. 